\title{
THE LANDSAT DATA CONTINUITY MISSION OPERATIONAL LAND IMAGER (OLI) RADIOMETRIC CALIBRATION
}

\author{
Brian L. Markham, Philip W. Dabney, \\ Jeanine E. Murphy-Morris, Jeffrey A. Pedelty \\ NASA/GSFC \\ Greenbelt, MD 20771 \\ brian.1.markham@nasa.gov \\ philip.w.dabney@nasa.gov \\ jeanine.e.murphy-morris@nasa.gov \\ jeffrey.a.pedelty@nasa.gov
}

Edward J. Knight, Geir Kvaran

Ball Aerospace \& Technology Corp. 1600 Commerce St.

Boulder, CO 80301

eknight@ball.com

gkvaran@ball.com
Julia A. Barsi

Science Systems Applications, Inc

GSFC

Greenbelt, MD 20771

julia.a.barsi@nasa.gov

\begin{abstract}
The Operational Land Imager (OLI) on the Landsat Data Continuity Mission (LDCM) has a comprehensive radiometric characterization and calibration program beginning with the instrument design, and extending through integration and test, on-orbit operations and science data processing. Key instrument design features for radiometric calibration include dual solar diffusers and multi-lamped on-board calibrators. The radiometric calibration transfer procedure from NIST standards has multiple checks on the radiometric scale throughout the process and uses a heliostat as part of the transfer to orbit of the radiometric calibration. On-orbit lunar imaging will be used to track the instruments stability and side slither maneuvers will be used in addition to the solar diffuser to flat field across the thousands of detectors per band. A Calibration Validation Team is continuously involved in the process from design to operations. This team uses an Image Assessment System (IAS), part of the ground system to characterize and calibrate the on-orbit data.
\end{abstract}

\section{INTRODUCTION}

The Landsat Data Continuity Mission (LDCM), a joint NASA and USGS mission, is on target for a December 2012 launch. Two sensors, the Operational Land Imager (OLI), being built by Ball Aerospace and Technology Corporation (BATC) under contract to NASA and the Thermal Infrared Sensor (TIRS), being built by NASA's Goddard Space Flight Center (GSFC), comprise the LDCM payload. The OLI covers the solar reflective part of the spectrum from visible to Short-Wave Infrared (SWIR); TIRS has two bands in the Long-Wave InfraRed (LWIR) emissive part of the spectrum. This paper will focus on the OLI sensor, as the TIRS radiometric calibration described in a companion paper [1].

\section{OLI DESIGN}

The OLI (Figure 1) is a $30 \mathrm{~m}$ ( $15 \mathrm{~m}$ Pan) Visible, Near-IR and SWIR push-broom sensor, covering spectral bands similar to ETM+ in this spectral region with additional bands at $443 \mathrm{~nm}$ and $1375 \mathrm{~nm}$, for coastal and aerosol applications and cirrus cloud detection, respectively [2]. The spectral bands are shown in Table 1 . The $15^{\circ}$ cross track field of view of the instrument is provided by a fourmirror anastigmatic telescope. The focal plane is made up of 14 Focal Plane Modules (FPM's), each containing the detectors (silicon for the VNIR bands and $\mathrm{HgCdTe}$ for the SWIR bands) and spectral band butcher-block filters for all the bands (Figure 2). There are thus approximately 6900 detectors per 30 -meter band and 13800 detectors for the Pan band.

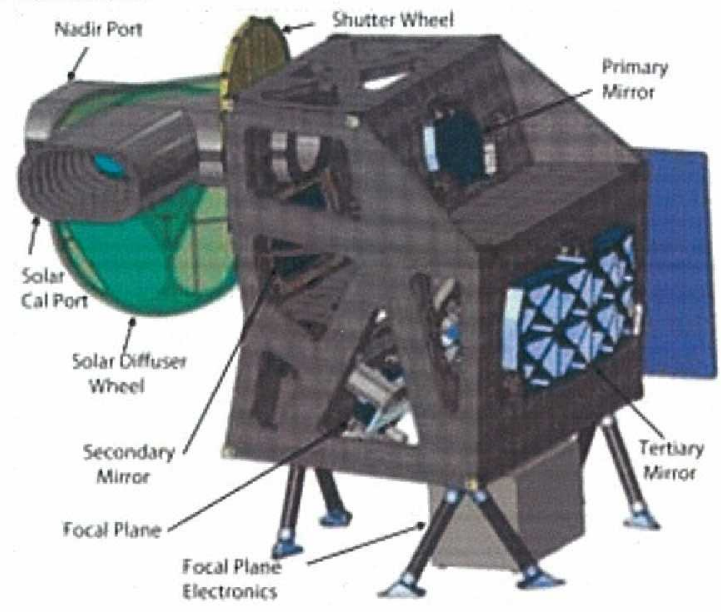

Figure 1: OLI Instrument; quaternary mirror not visible 
Table 1: OLI Nominal Spectral Bands

\begin{tabular}{|c|l|c|c|}
\hline$\#$ & \multicolumn{1}{|c|}{ Band } & $\begin{array}{c}\text { Lower Band } \\
\text { Edge (nm) }\end{array}$ & $\begin{array}{c}\text { Upper Band } \\
\text { Edge (nm) }\end{array}$ \\
\hline 1 & $\begin{array}{l}\text { Coastal } \\
\text { Aerosol }\end{array}$ & 433 & 453 \\
\hline 2 & Blue & 450 & 515 \\
\hline 3 & Green & 525 & 600 \\
\hline 4 & Red & 630 & 680 \\
\hline 5 & NIR & 845 & 885 \\
\hline 6 & SWIR 1 & 1560 & 1660 \\
\hline 7 & SWIR 2 & 2100 & 2300 \\
\hline 8 & Panchromatic & 500 & 680 \\
\hline 9 & Cirrus & 1360 & 1390 \\
\hline
\end{tabular}

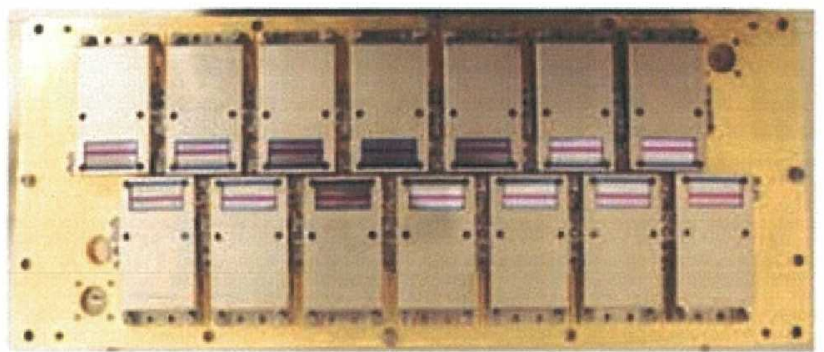

Figure 2. Flight OLI Focal Plane Assembly (FPA) showing 14 Focal Plane Modules (FPM's). Flight aperture mask not installed. FPM 219 is third from the right in the top row.

OLI data are quantized to 12 bits and the gains are set for each band so as not to saturate for $100 \%$ diffuse reflecting targets under the range of solar zenith angles to be present on orbit.

\section{ON-ORBIT CALIBRATION CAPABILITIES}

The OLI includes on-board radiometric calibration capabilities of: (1) dual full-aperture full-system Spectralon diffusers, designed to be used at different frequencies to aid in tracking the system and diffuser changes, and (2) multi-bulbed tungsten lamp assemblies, that illuminate the OLI detectors through the full optical system, similarly designed to be used at different frequencies to separate lamp and system changes. The lamp system also includes two built-in silicon monitor detectors, one for each lamp assembly. The primary solar diffuser will nominally be deployed every 8 days to track the calibration of the OLI sensor and perform detector-todetector normalization. The solar diffuser based calibration requires a spacecraft maneuver to point the OLI solar calibration aperture towards the sun. The pristine diffuser will be used on a less frequent basis, about every six months, as a check on the primary diffuser's degradation. The working lamp set will be used daily on the OLI, the reference lamp set approximately monthly and the pristine lamp set approximately twice a year. The LDCM operational concept also calls for the spacecraft to be maneuvered every lunar cycle to view the moon, providing a "known" stable source for tracking stability over the mission. A side-slither maneuver, where the spacecraft is rotated $90^{\circ}$ to align the detector rows with the velocity vector, is also planned. These data will provide an additional method to assess the detector-todetector radiometric normalization.

\section{PRE-LAUNCH SPECTRO-RADIOMETRIC CHARACTERIZATION AND CALIBRATION}

The spectral characterization of the OLI instrument is being performed at the component, focal plane module and full instrument levels. The components, which have all completed testing, include detector witness samples, spectral filters prior to dicing into flight filter sticks, the focal plane assembly window witness samples and telescope mirror witness samples. The FPM level tests, which are also complete, are specifically designed to characterize the spectral out-of-band response. The FPM level tests measure the spectral response of all the detectors by illuminating the full focal plane at approximately the correct cone angle. Figure 3 illustrates a typical NIR band response, a typical SWIR 1 band response and typical out-of-band to in-band response ratios. Note that the out-of-band response is well within requirements of less than $0.1 \%$ response away from the bandpass and total integrated response of less than $2 \%$ (Figure 3c). The FPM level tests indicate somewhat higher out-of-band response than the product of the filters and detectors: some of this is due to spectral stray light in the ground test equipment, e.g., the discontinuity in response in the NIR band at $450 \mathrm{~nm}$ is due to the removal of a long wavelength cut off (heat blocking) filter that blocks wavelengths above $700 \mathrm{~nm}$ and some of this is attributable to low level optical crosstalk, e.g., the response peak at $2200 \mathrm{~nm}$ for the SWIR 1 band is due to light going through the adjacent SWIR 2 filter, bouncing between the detector and filter assembly and reaching the SWIR 1 detectors. The upcoming system level tests are designed to characterize the in-band response. Here, a sampling of detectors is measured, circa $10 \%$.

An integrating sphere is used in the pre-launch radiance calibration of the OLI. The traceability of the calibration of this sphere will start with the 11" OLI transfer sphere directly calibrated at the NIST Facility for Spectroradiometric Calibration (FASCAL). While still at NIST, this OLI transfer sphere is checked by independently NIST calibrated University of Arizona (UAR VNIR transfer radiometer), NASA and NIST (Government Transfer Radiometers) radiometers (Figure 4). Also, the Ball Standard Radiometer (BSR), that has filters matching the OLI bands, views the sphere. 


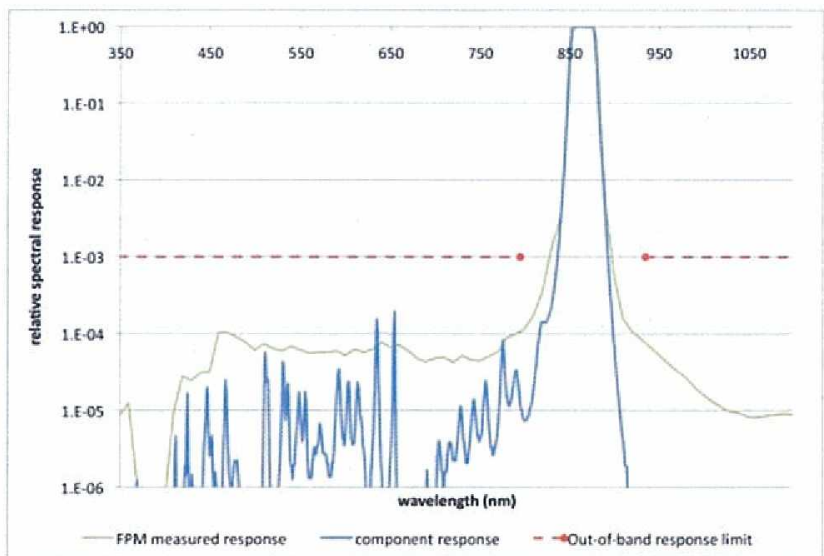

Figure 3a. NIR band average spectral response for FPM 219; component level measurements versus FPM level measurements.

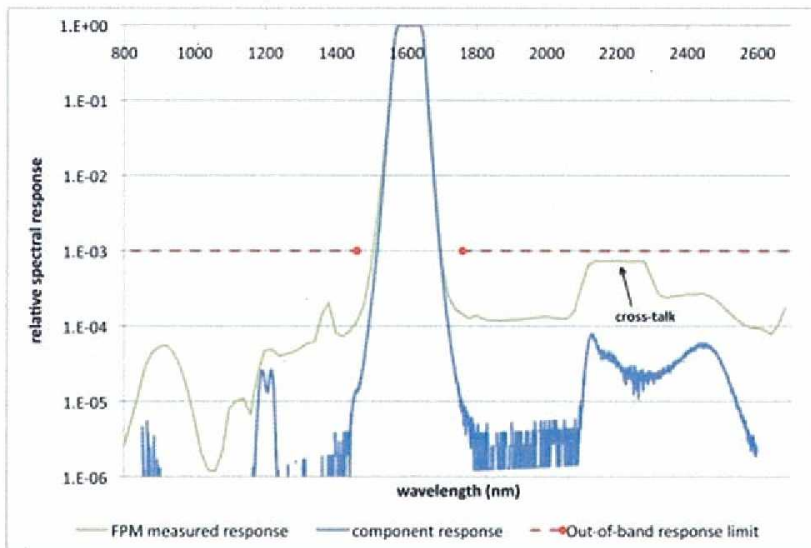

Figure $3 \mathrm{~b}$. SWIR 1 band average spectral response for FPM 219; component level measurements versus FPM level measurements.

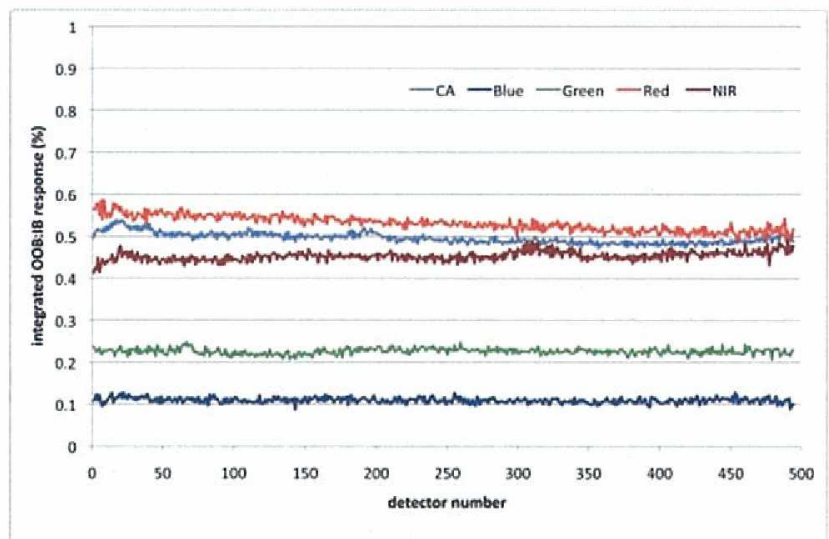

Figure 3c. OLI VNIR band out-of-band to in-band response ratio for FPM 219. Requirement is less than $2 \%$.

After return to Ball, the sphere is rechecked using its internal monitor and the BSR. At Ball, the radiance calibration is then transferred from the transfer sphere to a large integrating sphere dubbed the "Death Star Source (DSS)" using Ball's COTS Transfer Radiometer (CXR). The CXR is similar in design to the BSR, but also includes a spectrometer to aid in the calibration transfer. The DSS has internal radiance feedback control and monitors available for each OLI band. After the calibration transfer, the DSS radiance will be checked using the same transfer radiometers used to check the small transfer sphere. The OLI is then calibrated with the DSS with the OLI in thermal vacuum (T/V) and the DSS in ambient. An appropriately baffled window in the T/V chamber transmits the light to the OLI. The transmission of this window and baffling system is characterized by viewing the DSS with the CXR inside the T/V chamber without evacuation of the chamber. This radiance calibration will also be transferred to the OLI diffusers using a heliostat to provide a transfer-to-orbit standard. Inherent in the pre-launch radiometric calibration of the OLI is the need to apply a consistent calibration across the full $15^{\circ}$ Field of View (approximately 7000 detectors in the 30 meter bands). This is done, in part, by rotating the OLI instrument such that each detector views the same portion of the integrating sphere during calibration.

For the reflectance calibration of the OLI, a transfer calibration panel will be calibrated at NIST's STARR facility, including the recently available SWIR capability. The reflectance calibration will then be transferred to the flight diffusers.

Other pre-launch radiometric testing includes noise characterization (random, coherent, 1/f), linearity (both with radiance and integration time), stray light, bright target recovery and ghosting.

The ground system, being developed by USGS, includes an Image Assessment System (IAS), similar to Landsat7 's, to operationally monitor, characterize and update the calibrations of the two sensors. The IAS is described in a companion paper [3] and will include within its capabilities the processing of raw image data (Level 0R) to fully processed (Level 1) data as used in the Landsat Product Generation System (LPGS). The data from the internal lamps, the diffusers, the lunar and side slither acquisitions will be used to assess any changes to radiometric calibration parameters and update them as necessary. Particular emphasis is on the relative detectorto-detector radiometric normalization as the OLI has about 75000 detectors. In addition to the solar diffuser and side slither techniques, statistical treatment of the data from all Earth scenes (circa 400/day) will be also used to evaluate these relative gains. Additionally, IAS algorithms will characterize: (1) the noise performance of the OLI, e.g. overall Signal-to-Noise Ratio (SNR) using the diffuser and lamp signals, coherent, $1 / \mathrm{f}$, impulse and overall dark noise using the shutter data obtained before 
and after every acquisition interval, (2) the residual striping, banding and uniformity of the corrected imagery and (3) the stability of the instrument and data products.

As of this writing, the OLI flight telescope is complete and has been mated with the Engineering Development Unit (EDU) calibration assembly and EDU focal plane assembly to provide the EDU instrument. Stray light testing has been completed. EDU instrument spatial testing has begun with spectral and radiometric testing soon to follow. This EDU testing is designed to "wringout" the spatial, spectral and radiometric testing procedures. The small sphere source has been radiometrically calibrated at FASCAL and cross-checked with the various transfer radiometers. Flight instrument level testing is scheduled for Fall 2010. Revised spectral and radiometric performance estimates should be available based on the EDU testing.

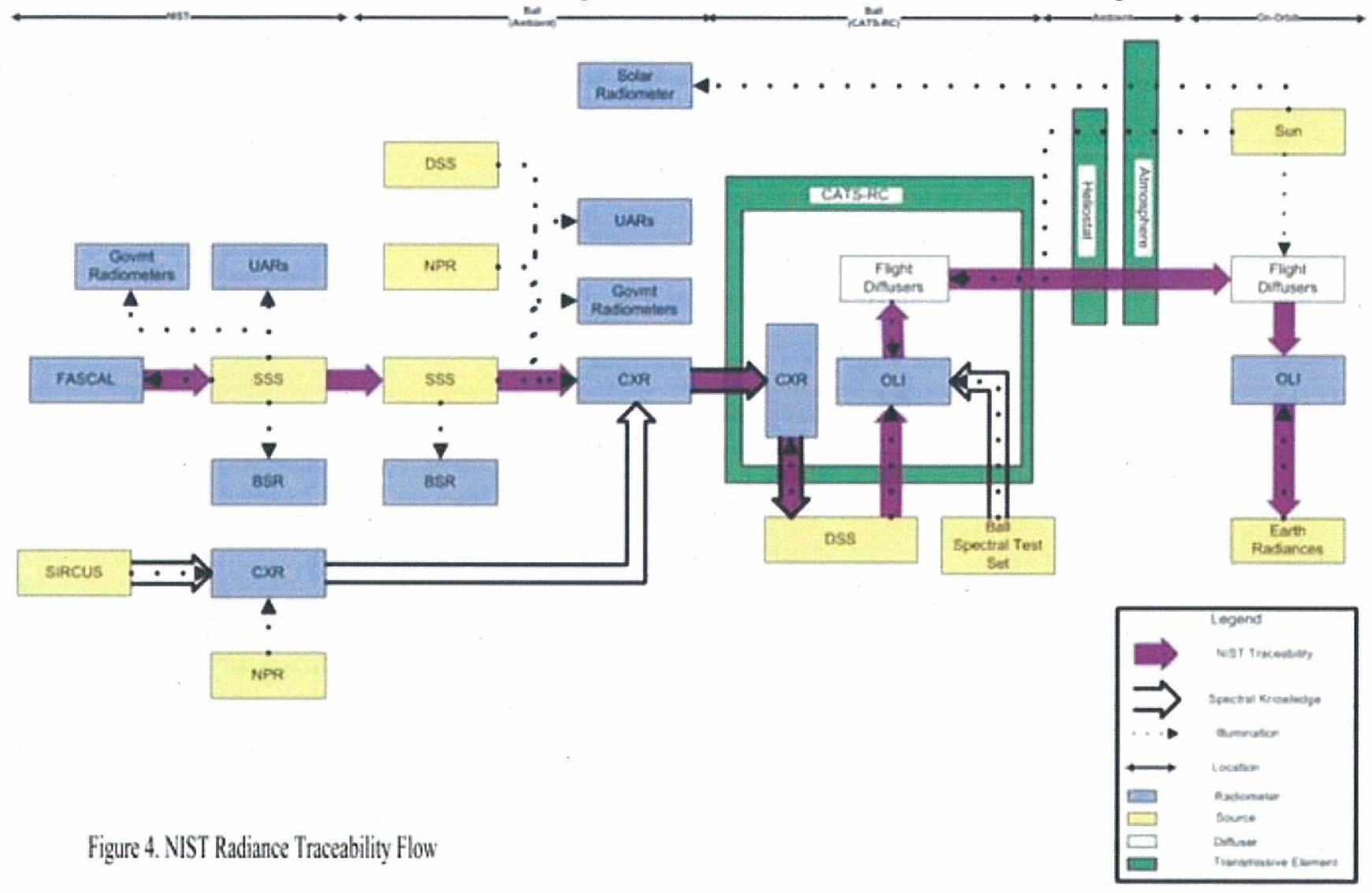

\section{ACKNOWLEDGEMENTS}

Ball has a large team of engineers dedicated to the prelaunch spectral and radiometric calibration of the OLI instrument. Beyond the authors, key personnel are Linda Fulton, Hansford Cutlip, Brent Canova, Amy Newbury, Kirk Lindahl, Sandra Collins and Holden Chase.

On the NASA Goddard side, Lawrence Ong, Robert Barnes, Edward Kaita and Raviv Levy are key contributors. At USGS/EROS, Ronald Morfitt, Esad Micijevic and Pasquale Scaramuzza lead the radiometry effort.

\section{REFERENCES}

[1] K. Thome, D. Reuter, C. Richardson, R. Smith. "Calibration of the Thermal Infrared Sensor on the
Landsat Data Continuity Mission," IEEE International Geoscience and Remote Sensing Symposium (IGARSS), JUL 25-30, 2010 Honolulu, HI (this issue).

[2] J. R. Irons and J. Murphy-Morris, "An Operational Land Imager for the Landsat Data Continuity Mission," IEEE International Geoscience and Remote Sensing Symposium (IGARSS), JUL 23-27, 2007 Barcelona, SPAIN, Pages: 2808-2810

[3] E. Micijevic and R. Morfitt, "Operational Calibration and Validation of the Landsat Data Continuity Mission (LDCM) Sensors using the Image Assessment System," IEEE International Geoscience and Remote Sensing Symposium (IGARSS), JUL 25-30, 2010 Honolulu, HI (this issue). 